\title{
Construction method of tailored facets for use in freeform reflectors design
}

\author{
Núria Tomás Corominas, Josep Arasa Martí
}

CD6. Universitat Politècnica de Catalunya Rambla Sant Nebridi 1008222 Terrassa

tomas@oo.upc.edu arasa@oo.upc.edu

\begin{abstract}
A construction method to obtain sets of tailored facets to be used as the initial configuration in the design of freeform reflector surfaces is presented. The construction strategy starts from a collection of flat elementary facets and achieves the initial configuration of the reflector surface by tailoring the facets according to their Bézier surfaces description. The aim of this research is to describe a procedure capable of generating the Bézier surfaces that define elementary tailored facets; these facets could then be used as initial configurations in the design of freeform reflectors. Though beyond the scope of this article, the facets could be used on a subsequent optimization process, i.e., the geometry of the elementary facets could be modified through a global optimization process taking into account all facets, in order to further improve the entire illumination system. Ray tracing is used to calculate the facet's geometry; it applies elementary merit functions to find the parameters defining the Bézier curve that best meets specifications in each elementary facet. This method is based on a sequential $2 \mathrm{D}$ projection strategy that employs different strategic planes for calculations. A method to build up the facet's geometry using a Bézier surface from 2D curves is provided. This approach will successfully generate a net of control points to describe a Bézier surface compatible with any standard optical optimization tool and suitable for use with CAD and other tools that represent solids.
\end{abstract}

Keywords: Non-Imaging Optics; Optical Design Freeform; Reflector; Faceted Reflector; Tailored Facet. 


\section{Introduction}

An optical design process is defined by a merit function, a quality criterion and an optimization algorithm. These are the basic elements that lead the optical system to the final required conditions (Turner and Garcia 2008).

Designers are aware that if the optimization process starts with a deficient initial configuration, the system will need a long time to reach a satisfactory result and the odds of success will be much lower. Consequently, a strategy successful in constructing a set of valid initial conditions constitutes an essential component of design processes (Ashdown, Brackett, and Sikkens 1994; Doyle, Corcoran, and Connell 1999).

A reflector design process is mainly based on energy contribution and its spatial and uniform distribution. Indeed, reflector designs are based on the relationship among three basic elements of optical design: source, reflector and target (Fournier, Cassarly, and Rolland 2010a; Fournier, Cassarly, and Rolland 2010b). Crucially, this relationship establishes the geometrical relations among the elements (Cassarly 2007).

When designing a reflector, three steps are essential to effectively attain the final reflector geometry:

- Segmentation: Source, target and reflector segmentation lead to reflector facets. The benefits of using segmented optical surfaces in non-imaging optics are widely documented in the literature (Cassarly et al. 2000; Leutz and Ries 2003; Pohl et al. 2003; Fournier, Cassarly, and Rolland 2010a; Fournier, Cassarly, and Rolland 2010b; Baeuerle et al. 2012). Faceting the reflector facilitates the required local adjustments of the geometric parameters of the facets so that the illumination goal at the target can be achieved. Different segmentation criteria must be defined to fulfil different criteria of energetic distribution; critically, the segmentation criteria must be flexible to adjust to any far field or near field situation. Several segmentation methods have been described (Timinger et al. 2000; Pohl et al. 2003; Sikkens and Nuyens 2003; Ries 2011).

- Position and orientation of every facet according to the proposed mapping. Various mapping techniques linking source, target and reflector facets have been described (Oliker and Newman 1993; Fournier, Cassarly, and Rolland 2010a; Baeuerle et al. 2012). Once the segmentation criteria based on the design boundary conditions have been defined, the facets' position in relation to pitch and tilt must be described.

- Tailoring: The local geometry of the facet's surface must be described in order to obtain the final goal of energetic distribution on the target(Cassarly et al. 2000; Timinger et al. 2000; Ries and Muschaweck 2001; Ries and Muschaweck 2002; Fournier, Cassarly, and Rolland 2010a; Fournier, Cassarly, and Rolland 2010b; Baeuerle et al. 2012).

These three steps define the process to build faceted optical surfaces from a geometrical structure. 
Tailoring, i.e., shaping the facets' surface so that illumination on the target meets a first approach of the required conditions, is the objective of our study. We propose a method to define a set of valid initial facets to be used in the optimization design process.

We have recently witnessed an explosion of methods based on heuristics rather than on the analytical search of exact solutions, including: soft computing methods, where we find evolutionary computing with genetic algorithms; neural computing, with algorithms inspired on the neural tissues distributed response; and KNS (Knowledge Based Systems) based on logics, probability and other variations (Kostrzewski et al. 1998; Gur, Mendlovic, and Zalevsky 2002).

Genetic algorithms have been proved useful in the area of optical design (Ashdown, Brackett, and Sikkens 1994; Cuevas et al. 2006; Fang and Tsai 2008; Wu et al. 2008; Xu 2011), where questions can be solved as optimization problems. Thus, from a set of initial conditions to develop a design, it is possible to calculate new successive approximations by modifying parameters with a similar role on the computational model than that of genes on the biological model.

To feed the optimization genetic algorithm, we need to obtain an initial diversified population of approximate solutions at low computational cost.

\section{Statement of the problem}

Before defining the method for tailoring the facets' surface, we should question the validity of searching for initial and final approximate solutions, since an exact theoretical solution (which can be unique) exists, made up of conical surfaces (Oliker 2003; Magarill 2011; Michaelis, Schreiber, and Braeuer 2011) in which source and target are assumed as points.

Two different explanations justify the build up of approximate solutions. Firstly, the design and mechanical constrains related to the optical surface. For instance, if an exact solution is to be found, the surface might be forced to pass through several points which disable the surface for being a solution of the optical design problem. Furthermore, the mathematical model used by CAD tools to shape the surface might not match with the surface solution built up with exact facets. In this case, the theoretical surface to reach compatible CAD tools must be adjusted, with the consequent loss of accuracy and widening the gap from the exact theoretical solution. The control of the optical behaviour is lost during the process of fitting surfaces to a theoretical model via mathematical methods. In an extreme case of fitting a high faceted multi-conical exact surface to a CAD compatible surface, the number of points of the adjusted surface which actually match the exact theoretical surface can decrease, with the subsequent loss of control of the real behaviour of light.

The second reason is related to the inner formulation of the design problem. What would happen if instead of a point energy distribution, which is used in the method based on exact theoretical solutions, we are given a general criterion described by a merit function? Instead of a discrete distribution of points as targets,

in most instances of real illumination problems, we are given a target illumination made of a general description of the desired light distribution corresponding to near field or far field distribution . This distribution can be expressed in terms of a merit function with different weights related to different 
zones of the targetConsequently, is it accurate to describe exact problems to be solved by exact techniques when the only actual information of the target is naturally given by a merit function? We believe that it would be preferable to work directly with merit functions than to generate a model of surface energy distributions to fit the requirement to the exact solution, thus a unique method would be valid for both types of light distribution, just adjusting the kind of merit function to the one related with the target

The aforementioned propositions are the cornerstone of the method presented in this article and based on two main elements:

The class of surfaces accepted as valid for designs are restricted to cubic Béziers. We have selected this class of functions because they are easy to operate and commonly used in CAD and in optical optimization tools (Yang et al. 2009; Jester, Menke, and Urban 2011; Michaelis, Schreiber, and Braeuer 2011). A calculation process computationally fast that converts the $3 \mathrm{D}$ problem to find cubic Bézier surfaces behaving as approximate solutions into a 2D problem to find Bézier curves on carefully chosen strategic planes.

With the application of this method we will be able to generate an initial population of approximate designs to feed the optimization algorithm. Each element of the population will be easy to achieve computationally and will be expressed by means of cubic Bézier surfaces, i.e., ready to be used by CAD tools with no need for adjustments or pre-processing.

The study of the error generated by this method has no relevance, since this assessment lies on the subsequent optimization algorithm. The calculation of the maximum possible error for a Bézier surface facet defined under certain conditions is beyond the scope of the current article.

\section{OBJECTIVE}

The objective of this research is to provide a method to calculate the local geometry of the reflector's facets as the first step towards a global procedure to obtain a diversified and approximate population of initial reflector designs able to feed algorithms for optimization. The method is focused on tailoring and calculates the geometrical parameters of the reflector facets as initial conditions. The facets are treated as singulars and are defined individually though under global conditions, thus describing freeform facets by means of quadratic Bézier surfaces that can be modified by a global optimization process (Doyle, Corcoran, and Connell 1999). Those conditions are derived from a previous segmentation and mapping process (Oliker and Newman 1993; Pohl et al. 2003; Fournier, Cassarly, and Rolland 2010a).Althought the mapping proces is outside the scope of the present papers, it might be taken into account that the success of the proposed method directly depends on the choice of mapping.

The tailored facets are calculated via a reduced number of rays valid for geometrical but not for photometric purposes. The use of limited numbers of rays entails low computational costs and is enough to obtain valid facet shapes.

Although optimization processes are not the objective of this research, we should mention that the global optimization process must take into account the real conditions and limitations of the fabrication process. The proposed method is focused on obtaining sets of tailored facets suitable for 
initial configurations. These configurations will lead to final sets of facets that can be freeform reflectors if they are somoothly connected, but in general, slope discontinuities in the final design will be present, resulting in faceted reflectors

\section{PROCEDURE}

This section describes the procedure to calculate the local geometry of the individual facets. We considered as valid the cubic Bézier surfaces, defined as $3 \times 3$ bicubic tensorial surfaces with $4 \times 4$ grid control points. The aim of this study is to describe a method capable of finding the surfaces' control points by means of a constructive method based on 2D Bézier curves calculated on meticulously chosen strategic planes; these Bézier curves are obtained under optical and energetic criteria through a local merit function and using a reduced number of rays. The initial condition to calculate the facets' local geometry originates from the four corners of a reflector whose outline has been previously set by the reflector segmentation process and oriented according to a predefined mapping. Though the four corners can be coplanar, they will usually form a hyperbolic paraboloid. Whether a plane or a hyperbolic paraboloid, the facet's surface can be improved by being defined as a cubic Bézier surface, i.e., a higher order surface with more parameters susceptible of adjustment. The calculation method enables the calculation of a Bézier surface that adjusts to the optical features described by the mapping algorithms, thus linking source, reflector and target.

Source and target are segmented during the segmentation process, where the central point of the segment source ( $\mathrm{S}$ ) and the central point of the segment target (T )are considered for optimization purposes (figure 1) Here, the pointS is considered as a point source emitting a fan of rays towards the facet. The point $\mathrm{T}$ is considered as a point target, around which the target is receiving the rays reflected on the reflector facet; a point to point relation can be defined among source and target. This method allows the consideration of different merit functions with regard to the distribution of light around $\mathrm{T}$.

This method is based on a 2D algorithm applied to few strategically chosen planes, to which we will refer as "calculation planes". A Bézier curve is found in each plane; a method to build up a valid surface is described from these few curves. The planes where the 2D algorithm will be applied must be carefully defined. Each plane is defined by two points of the surface's contour and the target. Since they are strategic planes, the surface is oriented for the source to be included in most of those planes; otherwise, an auxiliary source is used for calculation purposes to ensure that the target, source and two corners of the facet belong to the same plane, to which we can apply the 2D algorithm.

These conditions suffice to describe the facet as a Bézier surface ready to be used as initial conditions for a global optimization process.

The procedure is described in four separate steps:

- Ray tracing through $3^{\text {rd }}$ order 2D Bézier curves

- 2D optimization algorithm

- Selection of calculation planes

- Constructive method to build up cubic Bézier surfaces 


\subsection{Ray tracing through $3^{\text {rd }}$ order $2 D$ Bézier curves}

To describe the ray tracing method through a Bézier curve, the first step is to develop a 2D ray tracing algorithm (Canavesi, Cassarly, and Rolland 2012) through $3^{\text {rd }}$ order Bézier curves following equation (1):

$$
B(t)=(1-t)^{3} P_{0}+3(1-t)^{2} t P_{1}+3(1-t) t^{2} P_{2}+t^{3} P_{3}=t^{3} C_{1}+t^{2} C_{2}+t C_{3}+C_{4}
$$

where $P_{0}, P_{1}, P_{2}, P_{3}$ are the control points of the curve (figure 1a), $t \in(0,1)$ is the parameter of the Bézier curve and $C_{1}, C_{2}, C_{3}$ and $C_{4}$ are the following coefficients:

$$
\begin{aligned}
& C_{1}=\left(-P_{0}+3 P_{1}-3 P_{2}+P_{3}\right) \\
& C_{2}=3 P_{0}-6 P_{1}+3 P_{2} \\
& C_{3}=-3 P_{0}+3 P_{1} \\
& C_{4}=P_{0}
\end{aligned}
$$

Since the equation of a Bézier curve is a continuous function, the derivative can be found as

$$
B^{\prime}(t)=3 t^{2} C_{1}+2 t C_{2}+C_{3}
$$

The normal vector to the curve ( $\vec{N}$ ) can be calculated for any value of $t$. Thus, given a point source $\mathrm{S}$ and selecting equi-spaced values of $t$, a fan of rays covering the Bézier curve is defined (figure 1b), and at each selected impact point $B(t)$ an incident ray $\vec{R}$ is considered (figure 1a). The incident angle $\theta$ is calculated by means of $\vec{R}$ and $\vec{N}$ vector product:

$$
\sin \theta=\frac{|\vec{R} \wedge \vec{N}|}{|\vec{R}||\vec{N}|}
$$

The reflecting direction $\theta^{\prime}$ is calculated using Snell's law for reflectors: $\sin \theta=-\sin \theta^{\prime}$ (figure 1a) and a rotation of $\theta^{\prime}$ applied to $\vec{N}$ gives the direction of the reflected ray $\vec{R}^{\prime}$ : 


$$
\left(\begin{array}{cc}
\cos \theta^{\prime}-\sin \theta^{\prime} \\
\sin \theta^{\prime} & \cos \theta^{\prime}
\end{array}\right)\left(\begin{array}{l}
N x \\
N y
\end{array}\right)=\left(\begin{array}{l}
R^{\prime} x \\
R^{\prime} y
\end{array}\right)
$$

In this way, if the target is defined as a line by a point $\mathrm{T}$ and a direction $\vec{u}$, a collection of impact points coming from the reflected rays can be calculated (figure 1b).
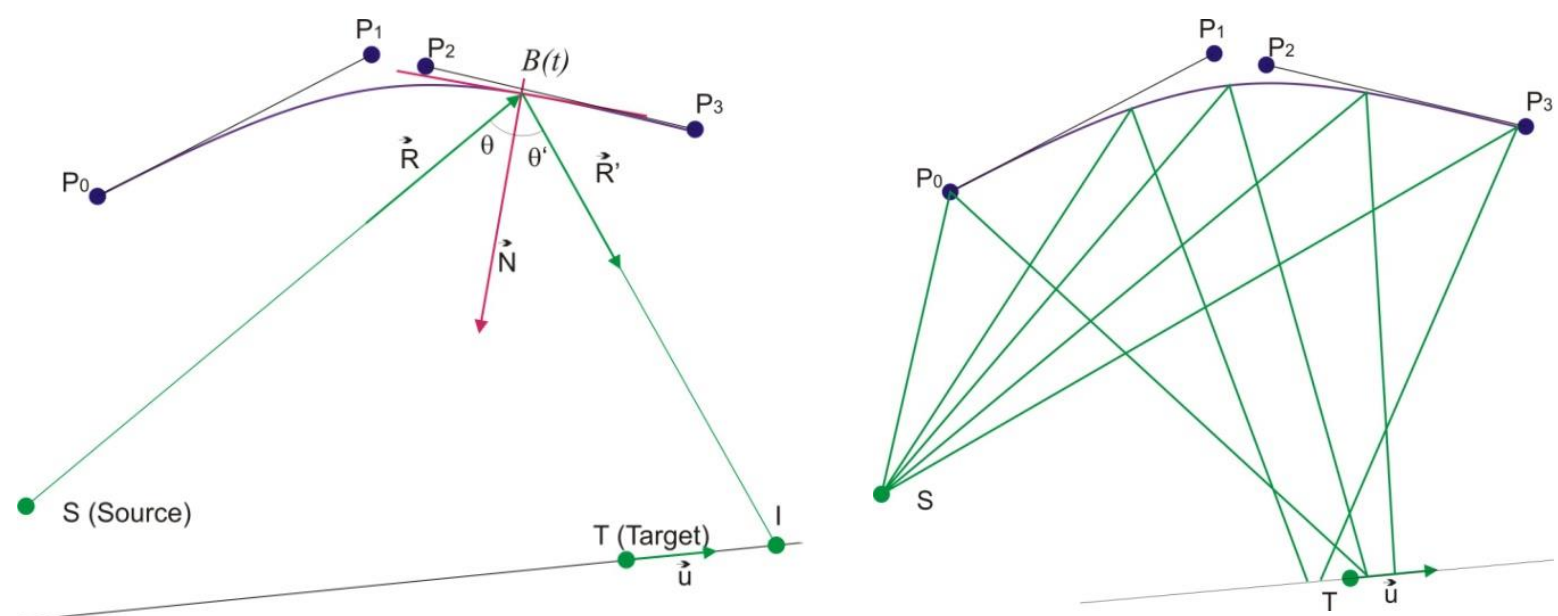

Figure 1 a) Bezier curve with its four control points, two of them $\left(P_{0}, P_{3}\right)$ lying on the curve, Snell law applied to a Bezier curve. Scheme of calculated values: impact point $\mathrm{B}(\mathrm{t}), \vec{N}$ as the normal direction of the curve in $\mathrm{B}(\mathrm{t})$, incident angle $\theta$ of the incident ray $\vec{R}$, reflecting angle $\theta^{\prime}$, direction of the reflected ray $\overrightarrow{R^{\prime}}$ and impact point I. Target lying on a line of direction $\vec{u} .2 \mathrm{~b}$ ) Ray tracing of several rays through a Bezier curve, from source $S$ to target $T$. A spot diagram around target $\mathrm{T}$ is obtained. Remind that point source and target are the central points of each elementary segment of source and target

The ray tracing method through a Bézier $2 \mathrm{D}$ curve has been described. This is the basis for the $2 \mathrm{D}$ optimization algorithm explained in the following section.

\subsection{Elementary $2 D$ optimization algorithm}

Once the basis of ray tracing through $3^{\text {rd }}$ order Bézier 2D curves has been established, the algorithm to obtain the best reflecting Bézier curve giving the required distribution of energy around the target point can be set using a merit function. The start up point are the four points lying on the calculation plane: source point $(\mathrm{S})$, target point $(\mathrm{T})$ and the two end points of the desired Bézier curve $\left(P_{0}, P_{3}\right)$. Using the local merit function, the goal is to find the two remaining Bézier control points $\left(P_{1}, P_{2}\right)$ in order to obtain the best Bézier curve for the defined reflecting purposes.

Some parameters must be defined a priori (figure 2): 
- Principal axis is the bisector of the lines SC and TC, $\mathrm{C}$ being the central point of the segment $\overrightarrow{P_{0} P_{3}}$.

- Shape parameter $\boldsymbol{L}$ is the distance between control points $P_{1}$ and $P_{2}$, and is related to the local curvature degree of the Bézier curve. A high value of the shape parameter leads to low curvatures, whereas a low value of the shape parameter leads to high curvatures.

- Progress factor $\boldsymbol{K}$ is a variable in the local optimization process. It indicates a path all along the principal axis

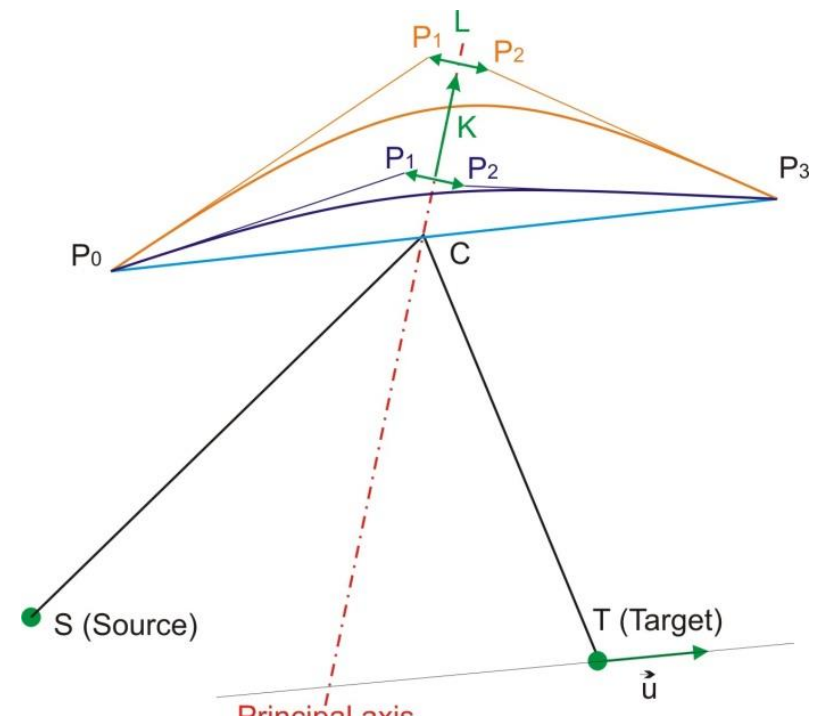

Figure 2 Two different curves obtained by displacing control points $\mathrm{P}_{1}$ and $\mathrm{P}_{2}$ along principal axis by a progress factor $K$. Distance between $P_{1}$ and $P_{2}$ is set by shape parameter $L$.

A segment centred on $\mathrm{C}$ and perpendicular to the principal axis is set up. The length of the segment is fixed by the shape parameter $L$ and is discretely moved through the axis following the progress factor $K$. In each progress step, the points $P_{1}$ and $P_{2}$ are set as the ends of the segment (Figure 2). Moving the segment along the principal axis, a collection of pairs $P_{1}$ and $P_{2}$ is obtained, thus defining a collection of Bézier curves.

As initial values, $\mathrm{L}$ and $\mathrm{K}$ are, respectively, $5 \%$ and $0.1 \%$ of the segment $\overrightarrow{P_{0} P_{3}}$, but further adjustments of those values can be done in order to improve results. For each Bézier curve, a fan of rays from the point source $S$ is pointed towards the curve, so that the reflecting rays and the impact diagram around the target are obtained for every defined Bézier curve. Thus, the best Bézier curve is selected using a weighted merit function of the vicinity of the impact points in relation to the point target $\mathrm{T}$. The process is computationally efficient due to the use of a short number of rays per fan.

The used merit functions are the following: 


$$
\begin{array}{r}
M F_{\text {conc }}=\frac{N}{\varepsilon}-\frac{N}{\sum_{n=1}^{N} D(n)+\varepsilon} \\
M F_{\exp }=\frac{1}{M} \sum_{i=1}^{M}\left(\frac{N}{\varepsilon}-\frac{N}{\sum_{n=1}^{N} D\left(n_{i}\right)+\varepsilon}\right)
\end{array}
$$

where $N$ is the number of rays used, $D(n)$ is the distance from each impact point to the target point (T) and $\varepsilon$ is a defined parameter to avoid singularities on the MF. On the other hand, $\mathrm{M}$ is the number of points used as control points within the desired area, which permits a regular distribution of light on the target area .

The merit function (9) has been based on the idea to concentrate the light around a target point, but other merit functions that distribute light around target, filling a specified area, have been defined, as shown on equation (10). The use of different merit functions would not prejudice the described method of finding Bézier surfaces.

\subsection{D: Selection of calculation planes of the facets}

This section describes the procedure to select six strategic planes $\left(\mathrm{SP}_{\mathrm{n}}\right)$ for the application of the 2D optimization algorithm. Departing from a facet reflector on which the four corners $R_{1}, R_{2}, R_{3}, R_{4}$ (figure 3) are set by the segmentation process, the objective is to find a suitable Bézier surface as initial condition for further optimization.
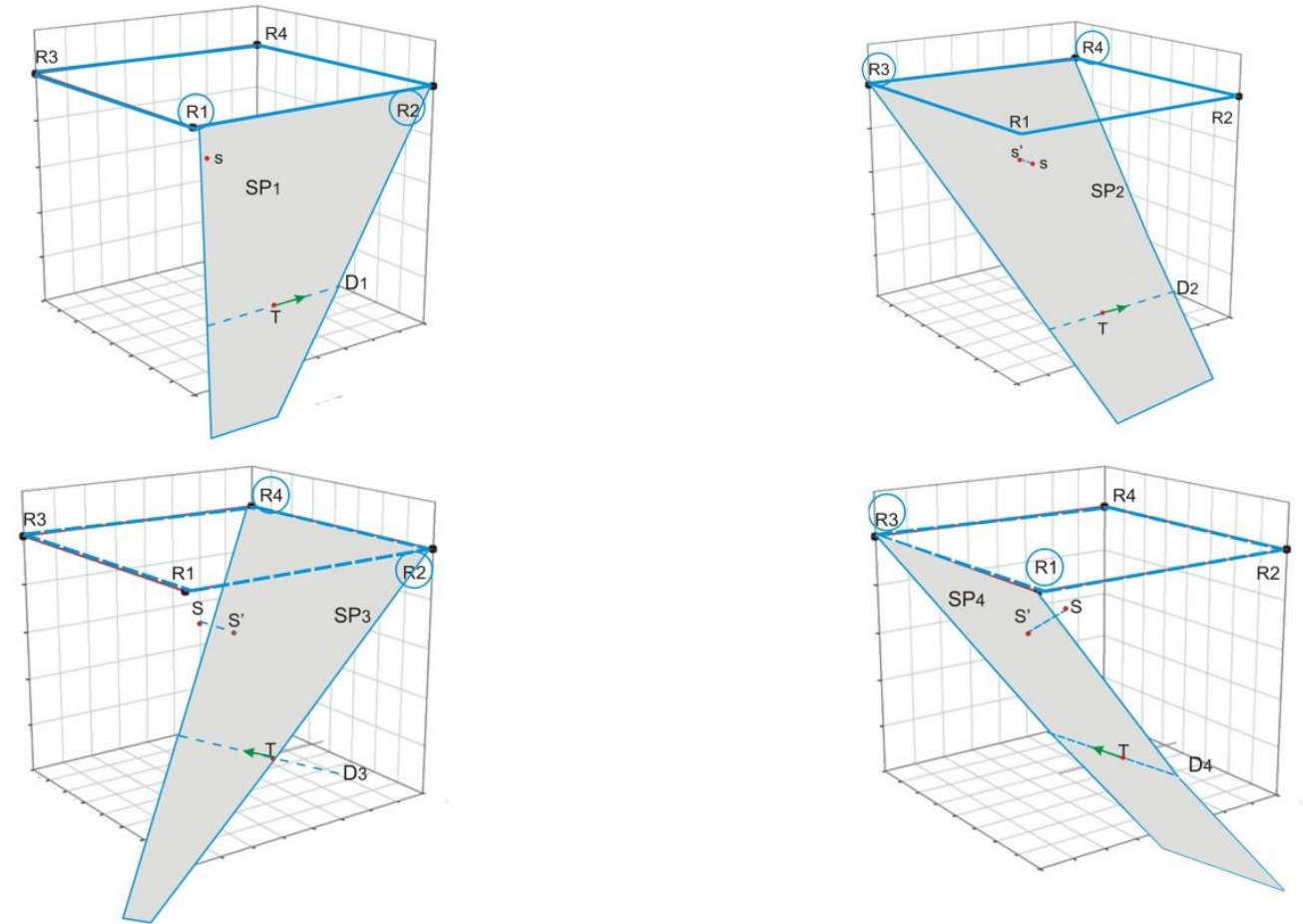
Figure 3 Initial flat reflector outlined by points R1, R2, R3 and R4. Source sited at S an target sited at $\mathrm{T} 3 \mathrm{a}$ ) Plane $\mathrm{SP}_{1}$ defined by points $\mathrm{R} 1, \mathrm{R} 2$ and $\mathrm{T}$ Line $\mathrm{D}_{1}$ contains $\mathrm{T}$ and is parallel to segment R1R2. 3b) Plane $\mathrm{SP}_{2}$ defined by points $\mathrm{R} 3, \mathrm{R} 4, \mathrm{~S}$. Line $\mathrm{D}_{2}$ contains $\mathrm{T}$ and is parallel to segment R3R4. 3c) Plane $\mathrm{SP}_{3}$ defined by points $\mathrm{R} 2, \mathrm{R} 4, \mathrm{~S}$. Line $\mathrm{D}_{3}$ contains $\mathrm{T}$ and is parallel to segment R2R4. S' is the projection of $\mathrm{S}$ over plane $\mathrm{SP}_{3} 3 \mathrm{~d}$ ) Plane $\mathrm{SP}_{4}$ defined by points $\mathrm{R} 1, \mathrm{R} 3, \mathrm{~S}$. Line $\mathrm{D}_{4}$ contains $\mathrm{T}$ and is parallel to segment $\mathrm{R} 1 \mathrm{R} 3$. $\mathrm{S}^{\prime}$ is the projection of $\mathrm{S}$ over plane $\mathrm{SP}_{4}$.

The first calculation plane $\mathrm{SP}_{1}$ is defined by two ends of the contour $\left(\mathrm{R}_{1}\right.$ and $\left.\mathrm{R}_{2}\right)$ and the target point $\mathrm{T}$ (figure $3 \mathrm{a}$ ). The spot diagram is calculated in a line $\mathrm{D}_{1}$ parallel to $\overline{R_{1} R_{2}}$ passing through the target $\mathrm{T}$. Three of the remaining calculation planes $\mathrm{SP}_{2}, \mathrm{SP}_{3}, \mathrm{SP}_{4}$ are defined following the same procedure, considering the other pairs of contour points as seen in figures $3 \mathrm{~b}, 3 \mathrm{c}$ and $3 \mathrm{~d}$. If the source $\mathrm{S}$ does not lie on $\mathrm{D}_{\mathrm{n}}$, as in segments $\overline{R_{2} R_{4}}$ and $\overline{R_{1} R_{3}}$ (figure $3 \mathrm{c}, 3 \mathrm{~d}$ ), a local source $\mathrm{S}_{\mathrm{n}}$ ' is defined by projecting the point $\mathrm{S}$ in the corresponding calculation plane and looking for the nearest distance from the original source $S$ to the plane.

The 2D algorithm defined in section 3.2 is applied to each calculation plane $\mathrm{SP}_{\mathrm{n}}$; as a result, a Bézier curve is found for each plane fulfilling the conditions of the merit function applied, that is minimizing the spot diagram around target $\mathrm{T}$ when using a concentrating merit function or achieving the size set by the expansive merit function . Consequently, four contour Bézier curves are obtained, two in the horizontal direction using planes $\mathrm{SP}_{1}$ and $\mathrm{SP}_{2}(\mathrm{QH} 0, \mathrm{QH} 3)$ and two in the vertical direction using planes $\mathrm{SP}_{3}$ and $\mathrm{SP}_{4}(\mathrm{QV} 0, \mathrm{QV} 3)$ (figure $\left.4 \mathrm{a}\right)$.

Two more auxiliary calculation planes, $\mathrm{SP}_{5}$ and $\mathrm{SP}_{6}$, are necessary to complete the build up of a Bézier surface. To describe them, two different values of parameter $t$ are chosen: $t_{1}$ and $t_{2}$. The pair of values of the Bézier curves (QV0 $\left.\left(\mathrm{t}_{1}\right), \mathrm{QV} 3\left(\mathrm{t}_{1}\right)\right)$ and $\left(\mathrm{QV0}\left(\mathrm{t}_{2}\right) \mathrm{QV} 3\left(\mathrm{t}_{2}\right)\right)$ define the ends of the auxiliary planes for the application of the 2D optimization algorithm. As a result, two more Bézier curves in the horizontal direction are obtained: QH1 and QH2, as seen in figures $4 \mathrm{a}$ and $4 \mathrm{~b}$.

The quartets of control points (11) of the four horizontal curves QH0, QH1, QH2, and QH3 are taken as the skeleton of the desired surface.

$$
Q=\left(\begin{array}{llll}
Q_{03} & Q_{13} & Q_{23} & Q_{33} \\
Q_{02} & Q_{12} & Q_{22} & Q_{32} \\
Q_{01} & Q_{11} & Q_{21} & Q_{31} \\
Q_{00} & Q_{10} & Q_{20} & Q_{30}
\end{array}\right)
$$



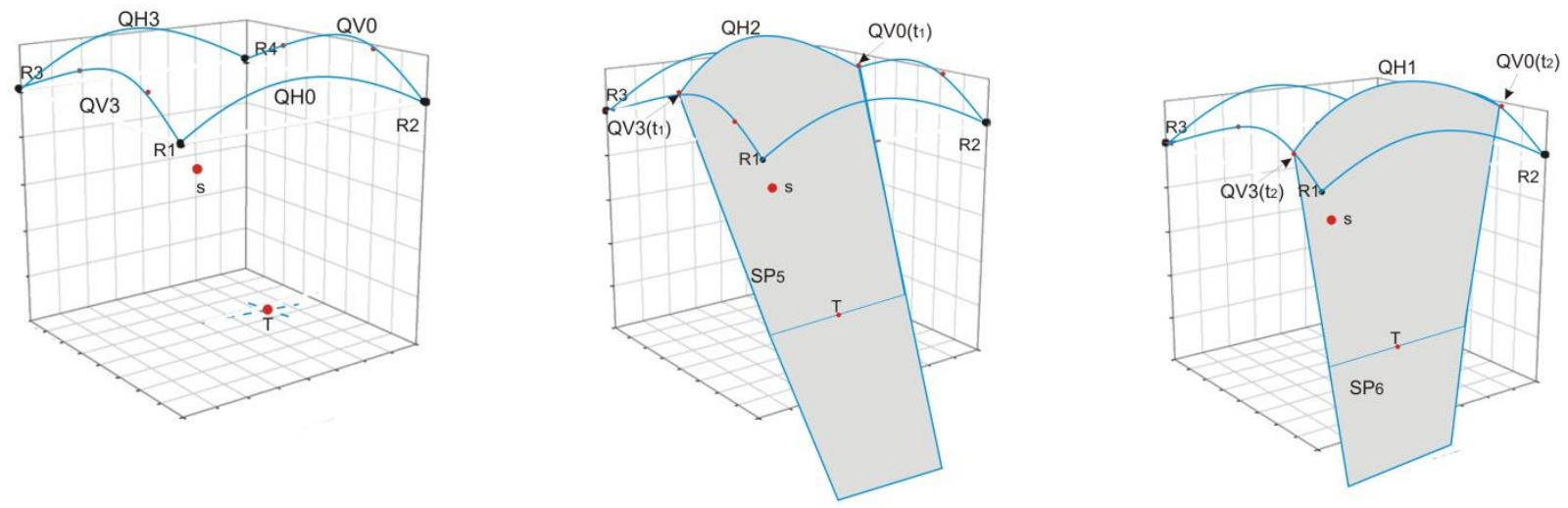

Figure 4 Construction of the two auxiliary planes. 4a) Bezier contour lines obtained in the four principal calculation planes. $4 \mathrm{~b}) \mathrm{QV0}\left(\mathrm{t}_{1}\right)$ and QV3 (t1) are the ends of the new auxiliary plane $\mathrm{SP}_{5}$ where 2D algorithm is applied to obtain the Bezier curve QH1. 4c) QV0 ( $\left.\mathrm{t}_{2}\right)$ and QV3 (t2) are the ends of the new auxiliary plane $\mathrm{SP}_{6}$ where $2 \mathrm{D}$ algorithm is applied to obtain the Bezier curve $\mathrm{QH} 2$

\subsection{Constructive method to build up cubic Bézier surfaces}

This section presents a method to characterize a cubic Bézier surface containing the four Bézier curves described in section $3.3(\mathrm{QH} 0, \mathrm{QH} 1, \mathrm{QH} 2, \mathrm{QH} 3)$. Some features of $3 \times 3$ tensor product Bézier surfaces are introduced prior to the description of the procedure for a better understanding of the Bézier reflective surfaces constructive method (Farin 2002).

The equation of a $3 \times 3$ Bézier surface is:

$$
P(s, t)=\sum_{i=0}^{3} \sum_{j=0}^{3} P_{i j} B_{i}^{3}(s) B_{j}^{3}(t)=\sum_{i=0}^{3}\left(\sum_{j=0}^{3} P_{i j} B_{i}^{3}(s)\right) B_{j}^{3}(t)
$$

where $P_{i j}$ are the control surface points, $P(s, t)$ are points of the surface, s and $t$ are the parameters of the surface, and $B_{i}^{3}(s)$ and $B_{j}^{3}(t)$ are Bernstein polynomials, of which the general formula is:

$$
B_{i}^{m}(s)=\left(\begin{array}{c}
m \\
i
\end{array}\right)(1-s)^{m-i} s^{i}
$$

The control net of a $3 \times 3$ Bézier surface is a $4 \times 4$ control points mesh, thus the control points $P_{i j}$ for a bicubic facet can be organized as follows: 


$$
P=\left[\begin{array}{llll}
P_{03} & P_{13} & P_{23} & P_{33} \\
P_{02} & P_{12} & P_{22} & P_{32} \\
P_{01} & P_{11} & P_{21} & P_{31} \\
P_{00} & P_{10} & P_{20} & P_{30}
\end{array}\right]
$$

The corresponding blending functions to express the surfaces in $s$ and $t$ parameters are:

$$
\left[\begin{array}{cccc}
(1-s)^{3} t^{3} & 3 s(1-s)^{2} t^{3} & 3 s^{2}(1-s) t^{3} & s^{3} t^{3} \\
(1-s)^{3} 3 t^{2}(1-t) & 3 s(1-s)^{2} 3 t^{2}(1-t) & 3 s^{2}(1-s) 3 t^{2}(1-t) & s^{3} 3 t^{2}(1-t) \\
(1-s)^{3} 3 t(1-t)^{2} & 3 s(1-s)^{2} 3 t(1-t)^{2} & 3 s^{2}(1-s) 3 t(1-t)^{2} & s^{3} 3 t(1-t)^{2} \\
(1-s)^{3}(1-t)^{3} & 3 s(1-s)^{2}(1-t)^{3} & 3 s^{2}(1-s)(1-t)^{3} & s^{3}(1-t)^{3}
\end{array}\right]
$$

where each row and column can be seen as an isoparametric Bézier curve. The whole surface can be thought of as a collection of isoparametric curves; the construction of such curves is described in terms of control curves. Control curves are auxiliary curves that do not lie on the surface. They consist of control points of the isoparametric curves of equation (15) and their own control points are surface control points $P_{i j}$ (equation (12)). Thus, if one of the parameters is fixed, for instance $s=c$, an isoparametric curve is obtained:

$$
P(c, t)=\sum_{j=0}^{3}\left[\sum_{i=0}^{3} P_{i j} B_{i}^{3}(c)\right] B_{j}^{3}(t)=\sum_{j=0}^{3} R_{j}(c) B_{j}^{3}(t)
$$

where $R_{j}(c)$ is the control curve that does not lie on the surface. The curves defined by control points $P_{i j} ; j=0,1,2,3$ (columns ) are the control curves steered by parameter $s$, whereas the curves defined by control points $P_{i j} ; i=0,1,2,3$ (rows) are the control curves steered by parameter $t$.

There are four special isoparametric curves that define the surface outline $P(0 ; t), P(1 ; t), P(s ; 0)$ and $P(s ; 1)$. They are Bézier curves defined by the columns or rows of corresponding control points; consequently, they also behave as control curves.

On the other hand, the strategic planes described in section 3.3 are set as slices of the solid angle formed by the target $\mathrm{T}$ and the corners of the facet (fig 3 ). The resulting Bézier curves corresponding to the facet contour are the isoparametric curves $P(0 ; t), P(1 ; t), P(s ; 0)$ and $P(s ; 1)$ described in equation (12) (Figure 3). These curves provide the 12 periphery control points depicted in bold in equation (17); therefore, the four central underlined control points must be given to obtain the complete mesh of control points. In general, the only control points lying on the surface are the four corners $\left(\mathrm{P}_{00}, \mathrm{P}_{03}, \mathrm{P}_{30}, \mathrm{P}_{33}\right)$ : 


$$
\boldsymbol{P}=\left[\begin{array}{llll}
\boldsymbol{P}_{03} & \boldsymbol{P}_{13} & \boldsymbol{P}_{23} & \boldsymbol{P}_{33} \\
\boldsymbol{P}_{02} & \frac{P_{12}}{P_{22}} & \boldsymbol{P}_{32} \\
\boldsymbol{P}_{01} & \frac{P_{11}}{P_{21}} & \boldsymbol{P}_{31} \\
\boldsymbol{P}_{00} & \boldsymbol{P}_{10} & \boldsymbol{P}_{20} & \boldsymbol{P}_{30}
\end{array}\right]
$$

Taking into account that the peripheral points of the matrix are both surface control points and isoparametric curve control points, only the four horizontal curves $\mathrm{QH}$ are needed to find the central control points of the surface. Thus, an equivalence of points, as shown in table 1 , is found when considering the Bézier control point matrix Q given in equation (11).

\begin{tabular}{|c|c|c|c|c|}
\hline $\begin{array}{c}\text { Isoparametric } \\
\text { curves }\end{array}$ & \multicolumn{4}{|c|}{$\begin{array}{l}\text { Bézier curves control points } Q_{i j} \\
\text { and the equivalence with surface control points } P_{i j}\end{array}$} \\
\hline $\mathrm{QH} 3$ with $t=1$ & $Q_{03}=P_{03}$ & $Q_{13}=P_{13}$ & $Q_{23}=P_{23}$ & $Q_{33}=P_{30}$ \\
\hline $\mathrm{QH} 2$ with $t=0.6$ & $Q_{02}=P_{02}$ & $Q_{12}$ & $Q_{22}$ & $Q_{32}=P_{31}$ \\
\hline QH1 with $t=0.3$ & $Q_{01}=P_{01}$ & $Q_{11}$ & $Q_{21}$ & $Q_{31}=P_{32}$ \\
\hline $\mathrm{QH} 0$ with $t=0$ & $Q_{00}=P_{00}$ & $Q_{10}=P_{10}$ & $Q_{20}=P_{20}$ & $Q_{30}=P_{30}$ \\
\hline
\end{tabular}

Table 1: Bézier curves control points $Q_{i j}$ and the equivalence with surface control points $P_{i j}$

The next step is to find the remaining surface control points $P_{12}, P_{22}, P_{11}, P_{21}$ using the $Q_{i j}$ curve control points through the construction of control curves (figure $5 \mathrm{c}$ ).

As stated previously, the auxiliary control curves are also Bézier curves, in this case defined by Bernstein polynomials. Taking the second and third control points of $\mathrm{QH}$ curves $\left(Q_{1 i}, Q_{2 i} i=0,1,2\right.$, $3)$, two control curves can be constructed. These two curves do not lie on the surface, but their control points are surface control points. By virtue of this property, the remaining surface control points can be next calculated (figure $5 \mathrm{~d}$ ). 

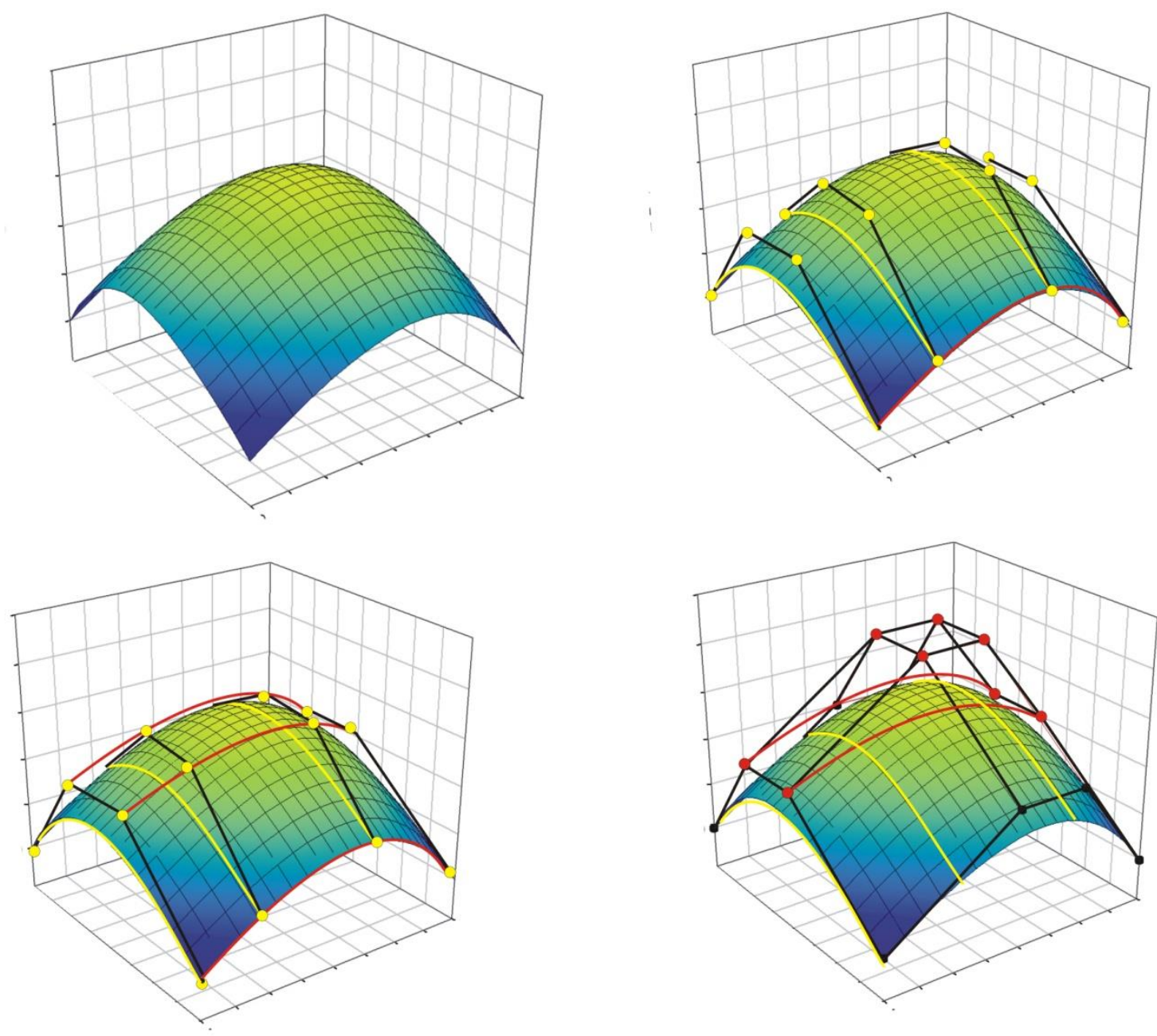

Figure 5 Construction method of a Bezier surface from Bezier curves 5a) Bezier surface generated by Bernstein polynomials as a tensorial product surface. 5b) Depicted in dashed lines QV vertical iso-parametric curves (fixed values: $t=0$ and $t=1$ ). Points of $Q V$ curves have been used as ends of QH curves. Control points of QV curves are not represented on the figure. Depicted in dotted lines four $\mathrm{QH}$ horizontal Bezier iso-parametric curves generated with fixed s values. Control points of QH Bezier curves depicted as empty points. All the curves lie on the surface. 5c) Depicted in dashed lines, two central control curves that does not lie on the surface. Control points of QH Bezier curves lie on control curves. 5d) Depicted as full points are the control points of the control curves. They are also control points of the surface. The four full points of the upper plane are the four control points calculated by the equation system.

An isoparametric curve is obtained by freezing parameters in equation (12). Thus for $s=c_{k}$, considering values as $c_{0}=0, c_{1}=0.3, c_{2}=0.6, c_{3}=1$, the coordinates of four curves can be obtained: 


$$
P\left(c_{k}, t\right)=\sum_{i=0}^{3}\left(\sum_{j=0}^{3} P_{i j} B_{i}^{3}\left(c_{k}\right)\right) B_{j}^{3}(t)
$$

On the other hand, four QH Bézier curves calculated via 2D algorithm of section 3.1 can be expressed following the equation:

$$
P\left(c_{k}, t\right)=\sum_{i=0}^{3} Q_{k j} B_{j}^{3}(t)
$$

Thus, from equations (18) and, the following equality can be stated:

$$
Q_{k j}=\sum_{i=0}^{3} P_{i j} B_{j}^{3}\left(c_{k}\right)
$$

The $Q_{k j}$ values are known as Bézier control points (table 1) and the specific $B_{j}^{3}\left(c_{k}\right)$ Bernstein coefficients can be calculated with equation (13) for $c_{0}=0, c_{1}=0.3, c_{2}=0.6, c_{3}=1$.

$$
B=\left(\begin{array}{llll}
B_{0}^{3}(0) & B_{0}^{3}(0.3) & B_{0}^{3}(0.6) & B_{0}^{3}(1) \\
B_{1}^{3}(0) & B_{1}^{3}(0.3) & B_{1}^{3}(0.6) & B_{1}^{3}(1) \\
B_{2}^{3}(0) & B_{2}^{3}(0.3) & B_{2}^{3}(0.6) & B_{2}^{3}(1) \\
B_{3}^{3}(0) & B_{3}^{3}(0.3) & B_{3}^{3}(0.6) & B_{3}^{3}(1)
\end{array}\right)=\left(\begin{array}{cccc}
1 & 0.343 & 0.064 & 0 \\
0 & 0.441 & 0.288 & 0 \\
0 & 0.189 & 0.432 & 0 \\
0 & 0.027 & 0.216 & 1
\end{array}\right)
$$

Equating term by term from equations (18) and (19), the following set of equations is obtained:

$$
c_{0}=0\left\{\begin{array}{l}
j=0 \rightarrow Q_{00}=P_{00} B_{0}^{3}(0)=\boldsymbol{P}_{00} \\
j=1 \rightarrow Q_{01}=P_{01} B_{0}^{3}(0)=\boldsymbol{P}_{01} \\
j=2 \rightarrow Q_{02}=P_{02} B_{0}^{3}(0)=\boldsymbol{P}_{02} \\
j=3 \rightarrow Q_{03}=P_{03} B_{0}^{3}(0)=\boldsymbol{P}_{03}
\end{array}\right.
$$




$$
\begin{aligned}
& c_{1}=0.3\left\{\begin{array}{l}
j=0 \rightarrow Q_{10}=\boldsymbol{P}_{00} B_{0}^{3}\left(c_{1}\right)+P_{10} B_{1}^{3}\left(c_{1}\right)+P_{20} B_{2}^{3}\left(c_{1}\right)+\boldsymbol{P}_{30} B_{3}^{3}\left(c_{1}\right) \\
j=1 \rightarrow Q_{11}=\boldsymbol{P}_{01} B_{0}^{3}\left(c_{1}\right)+P_{11} B_{1}^{3}\left(c_{1}\right)+P_{21} B_{2}^{3}\left(c_{1}\right)+\boldsymbol{P}_{31} B_{3}^{3}\left(c_{1}\right) \\
j=2 \rightarrow Q_{12}=\boldsymbol{P}_{02} B_{0}^{3}\left(c_{1}\right)+P_{12} B_{1}^{3}\left(c_{1}\right)+P_{22} B_{2}^{3}\left(c_{1}\right)+\boldsymbol{P}_{32} B_{3}^{3}\left(c_{1}\right) \\
j=3 \rightarrow Q_{13}=\boldsymbol{P}_{03} B_{0}^{3}\left(c_{1}\right)+P_{13} B_{1}^{3}\left(c_{1}\right)+P_{23} B_{2}^{3}\left(c_{1}\right)+\boldsymbol{P}_{33} B_{3}^{3}\left(c_{1}\right)
\end{array}\right. \\
& c_{2}=0.6\left\{\begin{array}{l}
j=0 \rightarrow Q_{20}=\boldsymbol{P}_{00} B_{0}^{3}\left(c_{2}\right)+P_{10} B_{1}^{3}\left(c_{2}\right)+P_{20} B_{2}^{3}\left(c_{2}\right)+\boldsymbol{P}_{30} B_{3}^{3}\left(c_{2}\right) \\
j=1 \rightarrow Q_{21}=\boldsymbol{P}_{01} B_{0}^{3}\left(c_{2}\right)+P_{11} B_{1}^{3}\left(c_{2}\right)+P_{21} B_{2}^{3}\left(c_{2}\right)+\boldsymbol{P}_{31} B_{3}^{3}\left(c_{2}\right) \\
j=2 \rightarrow Q_{22}=\boldsymbol{P}_{02} B_{0}^{3}\left(c_{2}\right)+P_{12} B_{1}^{3}\left(c_{2}\right)+P_{22} B_{2}^{3}\left(c_{2}\right)+\boldsymbol{P}_{32} B_{3}^{3}\left(c_{2}\right) \\
j=3 \rightarrow Q_{23}=\boldsymbol{P}_{03} B_{0}^{3}\left(c_{2}\right)+P_{13} B_{1}^{3}\left(c_{2}\right)+P_{23} B_{2}^{3}\left(c_{2}\right)+\boldsymbol{P}_{33} B_{3}^{3}\left(c_{2}\right)
\end{array}\right. \\
& c_{3}=1\left\{\begin{array}{l}
j=0 \rightarrow Q_{30}=P_{30} B_{3}^{3}(1)=\boldsymbol{P}_{30} \\
j=1 \rightarrow Q_{31}=P_{31} B_{3}^{3}(1)=\boldsymbol{P}_{31} \\
j=2 \rightarrow Q_{32}=P_{32} B_{3}^{3}(1)=\boldsymbol{P}_{32} \\
j=3 \rightarrow Q_{33}=P_{33} B_{3}^{3}(1)=\boldsymbol{P}_{33}
\end{array}\right.
\end{aligned}
$$

where the bold $P_{i j}$ points are the required points, whereas the underlined $P_{i j}$ points are known Bézier surface control points.

This can be expressed as matrix products:

$$
B S * P S=Q S-B_{c} * P_{c}
$$

where

$$
B S=\left(\begin{array}{ll}
B_{1}^{3}\left(c_{1}\right) & B_{2}^{3}\left(c_{1}\right) \\
B_{1}^{3}\left(c_{2}\right) & B_{2}^{3}\left(c_{2}\right)
\end{array}\right)
$$




$$
\begin{aligned}
P S & =\left(\begin{array}{llll}
\underline{P_{10}} & \underline{P_{11}} & \underline{P_{12}} & \underline{P_{13}} \\
\underline{P_{20}} & \underline{P_{21}} & \underline{P_{22}} & \underline{P_{23}}
\end{array}\right) \\
Q S & =\left(\begin{array}{llll}
Q_{10} & Q_{11} & Q_{12} & Q_{13} \\
Q_{20} & Q_{21} & Q_{22} & Q_{23}
\end{array}\right) \\
B_{c} & =\left(\begin{array}{lll}
B_{0}^{3}\left(c_{1}\right) & B_{3}^{3}\left(c_{1}\right) \\
B_{0}^{3}\left(c_{2}\right) & B_{3}^{3}\left(c_{2}\right)
\end{array}\right) \\
P_{c} & =\left(\begin{array}{llll}
\boldsymbol{P}_{00} & \boldsymbol{P}_{01} & \boldsymbol{P}_{02} & \boldsymbol{P}_{03} \\
\boldsymbol{P}_{30} & \boldsymbol{P}_{31} & \boldsymbol{P}_{32} & \boldsymbol{P}_{33}
\end{array}\right)
\end{aligned}
$$

Thus, the Bézier surface control points $P_{12}, P_{22}, P_{11}$ and $P_{21}$ can be calculated solving the following matrix:

$$
P S=B S^{-1}\left[Q S-B_{c} * P_{c}\right]
$$

to finally obtain all the necessary control points to build up the surface using equation (26).

\section{RESULTS}

For illustration purposes, the experiment's framework has been taken from a standard street lamp ). However, any other kind of lamp or illumination system could have been selected to demonstrate the viability of the method. The example shows how to set the initial surface parameters of a single facet and how the method is able to generate both a regular expanded patch of light and a concentrated spot around the target point. The surface is then described as a Bezier surface, and the selection criterion to choose proper parameters will be the amount of energy distribution around the defined target point.

We start from a reflecting surface divided into facets that have to be shaped. The contour of each facet in the space and the assignation of a target point to each facet are defined by the previous segmentation and mapping processes.

To exemplify how to calculate the local parameters of the Bezier surface, only one facet of the whole reflector is shown. The calculation is performed considering a point source with an arbitrary flux so that the parameter chosen to select the right Bezier coefficients is the normalized density of energy for individual facet.

To show the capability and flexibility of the method, a single facet shaped for different working conditions is calculated. This facet has been directed at different target points and in each of these conditions the process to obtain different Bezier surfaces has been led by the use of concentrating or expanding functions. 

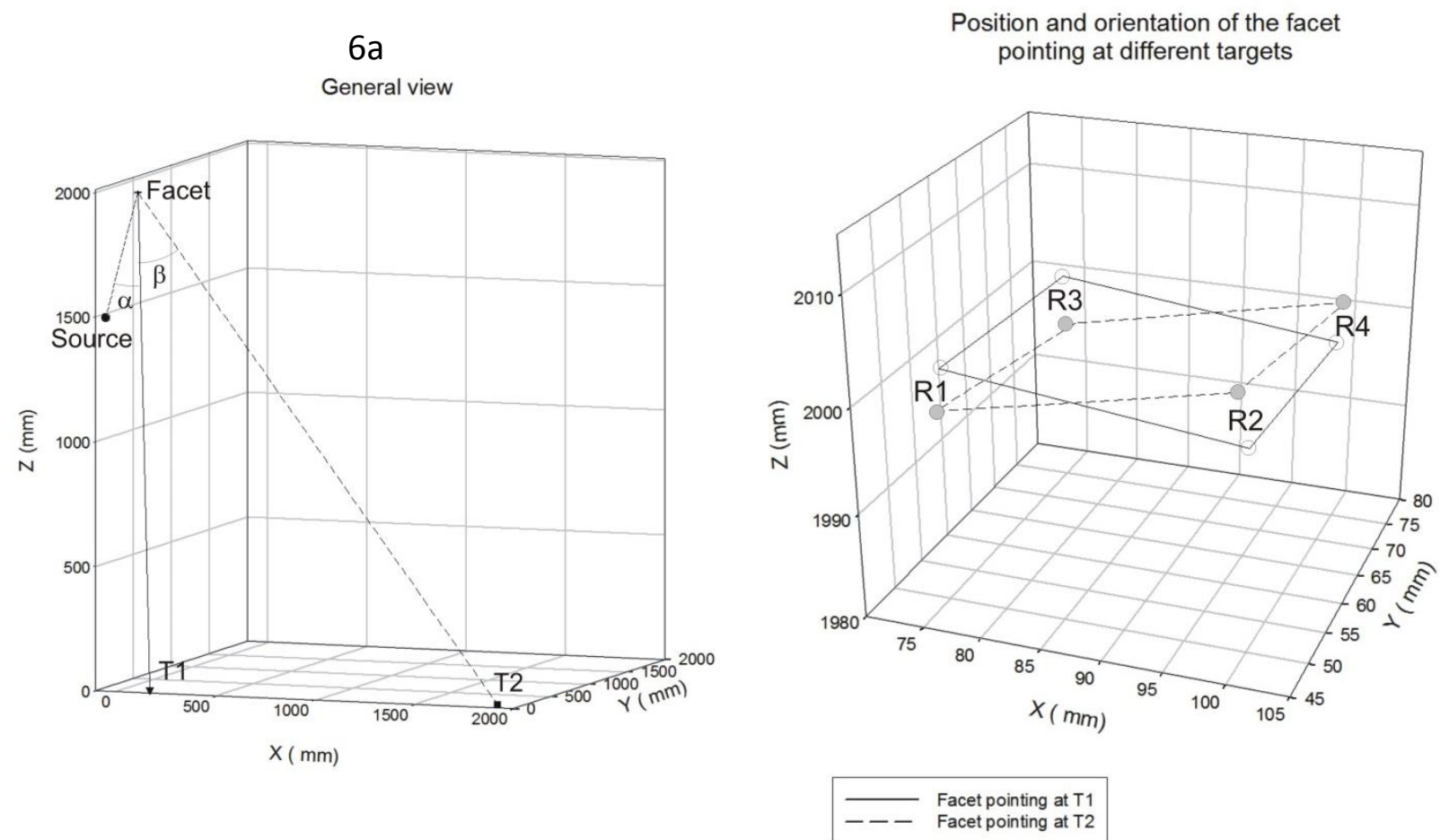

Figure 6a: Schematic view of the initial position of the facets, position of the source and corresponding target points $\mathrm{T} 1$ and $\mathrm{T} 2$. The rays hitting the centre of the facet and pointing to corresponding target $\mathrm{T} 1$ and target $\mathrm{T} 2$ are showed. Target $\mathrm{T} 1$ is at $\square \square \square 1^{\circ} 5^{\prime}$ from the vertical axis and $\mathrm{T} 2$ is at $\square \square \square 41^{\circ} 47^{\prime}$ from the vertical axis

Figure 6b: detailed view of the position of the facet. The facet when pointing at target T1 is represented in continuous contour and the facet when pointing at target $\mathrm{T} 2$ is represented in dotted contour.

Once the facet has been selected, two different target points are chosen (fig 6a) and the facet is next oriented towards each situation ( figure 6b). The input data for the example are the four corners of the corresponding facet reflector $\left(R_{1}, R_{2}, R_{3}, R_{4}\right)$, the position of the assigned targets $T_{1}$ and $T_{2}$, and the position of the point source $S$ (figure 6). The coordinates of the elements and all the distances can be found in table 1. For each target, two Bezier surfaces are designed, one to obtain a regular expanded patch of light, and the other one to obtain a concentrated spot around the target point, thus four different surfaces are obtained as a result of the implementation of the procedure. These surfaces are then incorporated into optical simulation software in order to achieve the illumination distribution. These results are next compared with those obtained with a flat reference surface pointing at the two different target points T1, and T2 (figures 7,10). On the expanded patch function example, the aim was to create a regular distribution of light around target $T$ with a size of $400 \times 400$ $\mathrm{mm}$ (figures 8,11). With regard to the concentrated spot function, the aim was to concentrate the light around the target point with the smallest possible spotlight (figures 9, 12). 
The example shows that once the outline of a facet is provided, this method is able to find the suitable Bezier surface to achieve the proposed illumination goals. The illumination goals can be expansive or concentrating, and in both cases better results are achieved if compared with an original flat facet. The results also prove that the method is valid for different positions of the target. Accordingly, if a single facet can be adapted to any illumination condition and to different positions of the target, the method could be applied to any single facet of the whole reflector.

Table 2. Position and size of the facet, source and targets

\begin{tabular}{|l|c|c|}
\hline \multicolumn{3}{|c|}{$\begin{array}{c}\text { Facet Contour coordinates } \\
\text { (all values in mm) }\end{array}$} \\
\hline Facet size & \multicolumn{2}{|c|}{$25 \times 25$} \\
\hline Source position & Target T1 & Target T2 \\
\hline & $(125,125,0)$ & $(1875,1125)$ \\
\hline Target position & $(75.1,49.9,2002.1)$ & $(74.5,50.1,1998.0)$ \\
\hline R1 & $(99.99,50.0,1999.0)$ & $(98.8,49.9,2003.8)$ \\
\hline R2 & $(75.1,74.9,2001.0)$ & $(76.1,75.0,1996.3)$ \\
\hline R3 & $(99.9,75.0,1997.8)$ & $(100.5,74.9,2001.9)$ \\
\hline R4 & \multicolumn{2}{|c|}{ ( 1900$)$} \\
\hline
\end{tabular}

In the following pictures the irradiance is normalized at 1 and depicted on a grey scale, where white is no light, black is the maximum 
$7 a$

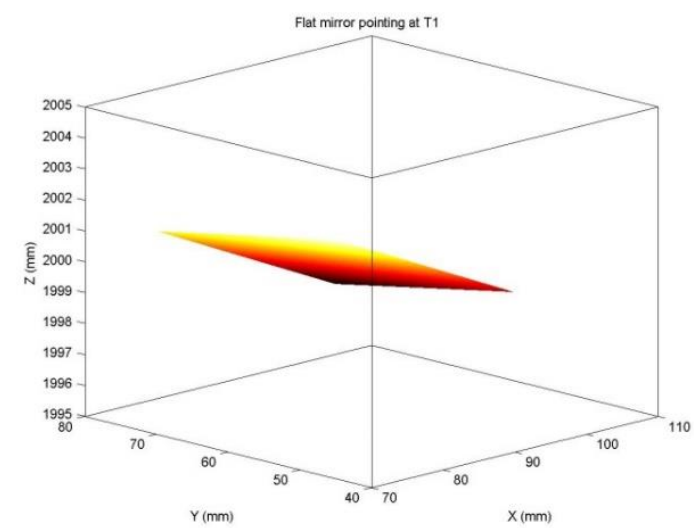

7c

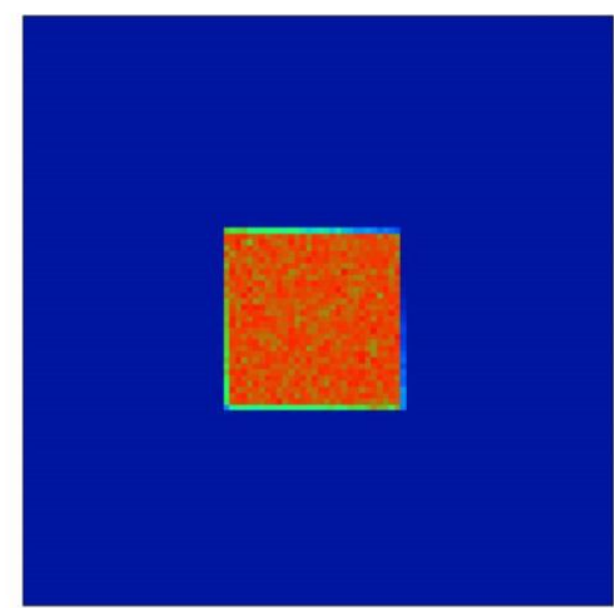

$7 b$

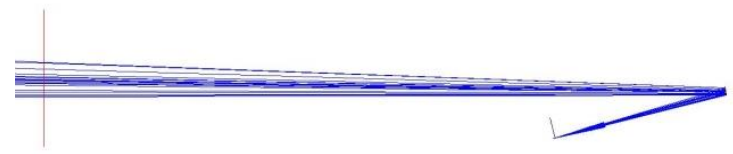

$7 d$

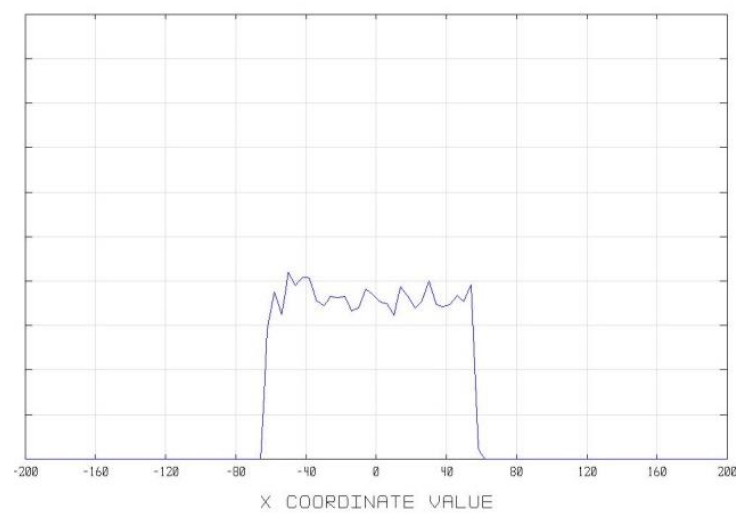

Figure 7 :.Flat reference mirror pointing at target T1.

Figure 7a: Surface of the reference flat mirror.

Figure $7 \mathrm{~b}:$ Zemax layout

Figure 7c: Image obtained with Zemax simulation using $10^{6}$ rays. Size of the spot diagram $128 \times 129 \mathrm{~mm}$.

Figures 7c and 7d: $X$ irradiance section.

All distances in $\mathrm{mm}$. Irradiance normalized at 1 
$8 a$

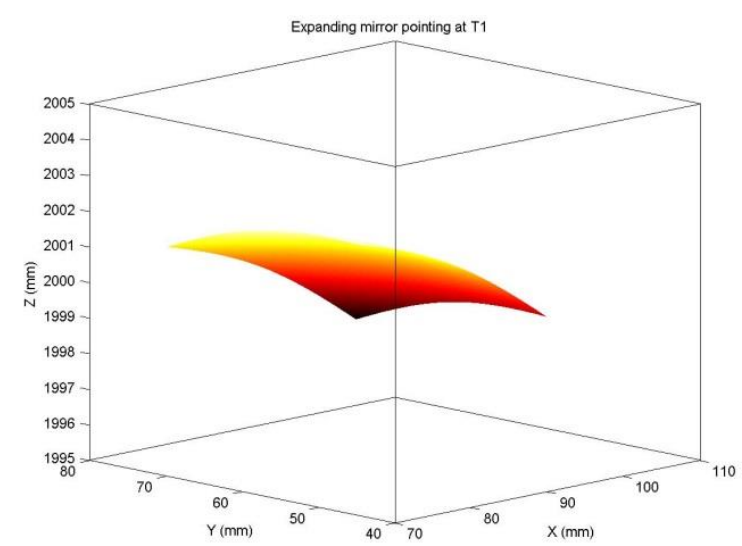

$8 c$

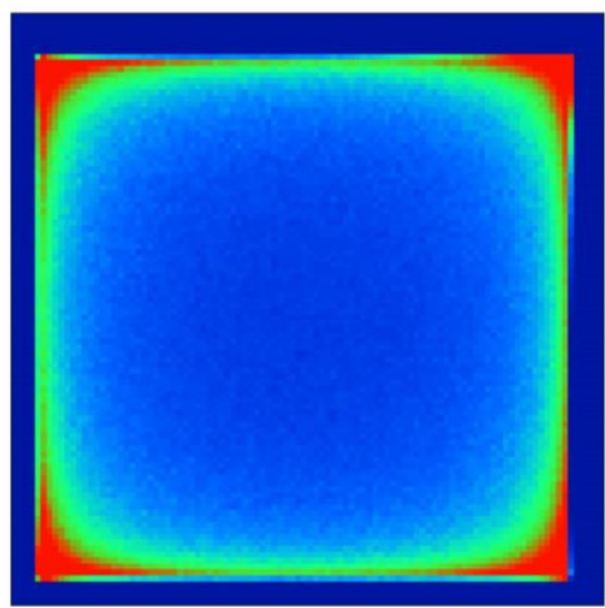

$8 b$

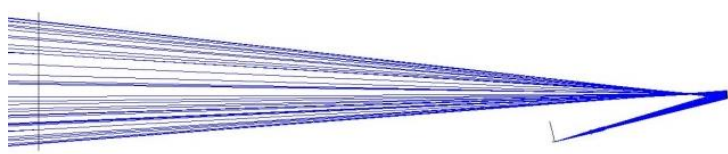

L.

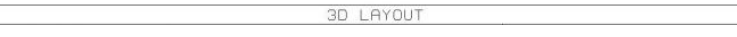

$8 d$

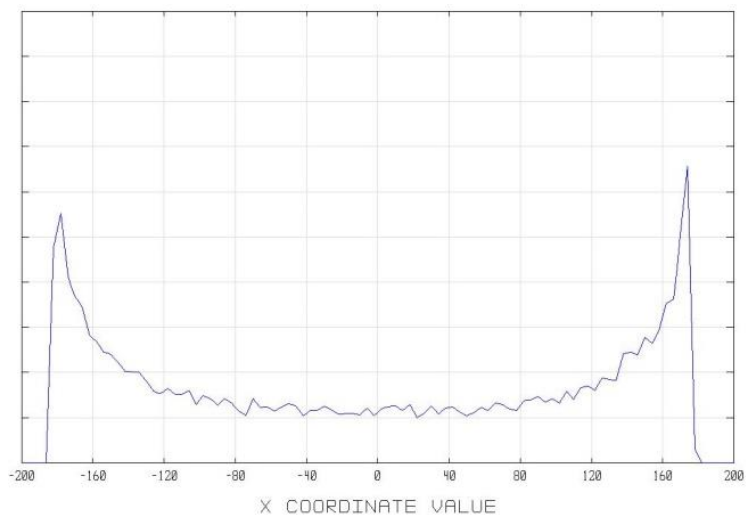

Figure 8: Mirror obtained with an expansive merit function aiming at a 400x400 $\mathrm{mm}$ squared patch illumination around target $\mathrm{T} 1$.

Figure 8a:Surface obtained after an elementary optimization process .

Figure 8b : Zemax layoutFigure 8c: Image obtained with Zemax simulation using $10^{6}$ ray. Size of the spot diagram $371 \times 363 \mathrm{~mm}$. Obtained size is $10 \%$ smallerthan expected.

Figures $8 \mathrm{~d}$ : $\mathrm{X}$ irradiance section.

All distances in mm. Irradiance normalized at 1 
$9 a$

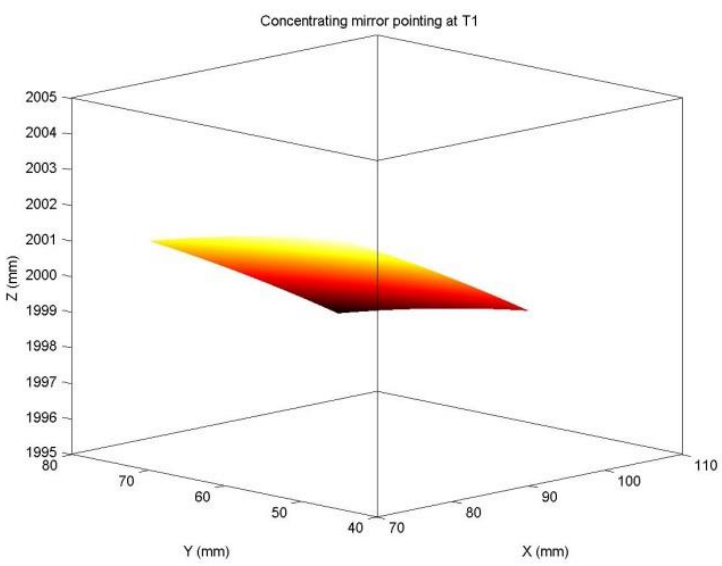

$9 c$

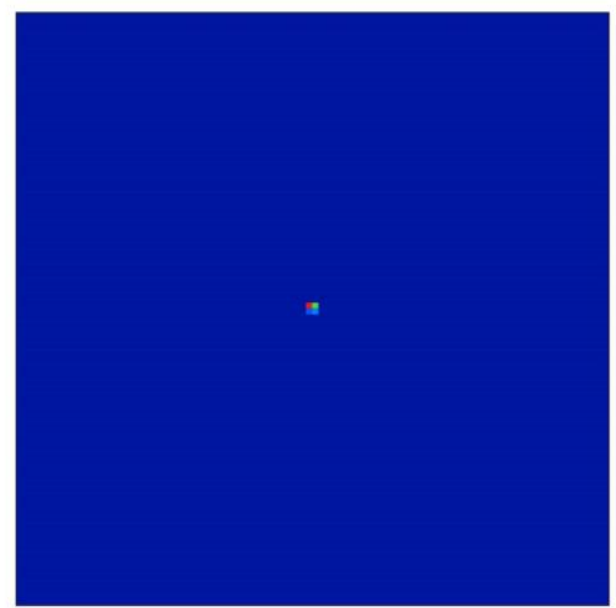

$9 b$

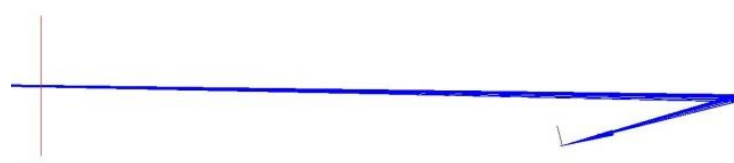

L.

$9 d$

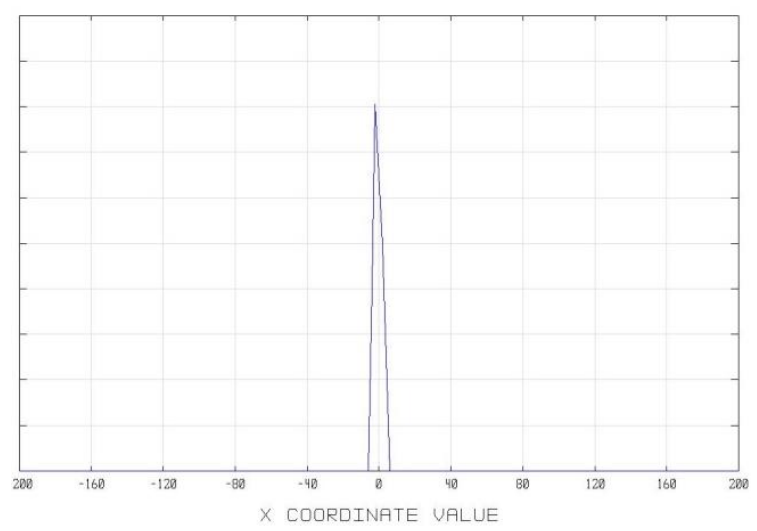

Figure 9 : Mirror obtained with a concentration merit function aiming to obtain the minimum spot around around target $\mathrm{T} 1$.

Figure 9a: Surface obtained after an elementary optimization process.

Figure 9b : Zemax layout

Figure 9c: Image obtained with Zemax simulation using $10^{6}$ ray. Size of the spot diagram $6.2 \times 8.5 \mathrm{~mm}$. Reduction size compared with the reference flat mirror is $93.5 \%$.

Figures 9d: $X$ irradiance section.

All distances in mm. Irradiance normalized at 1 
$10 \mathrm{a}$

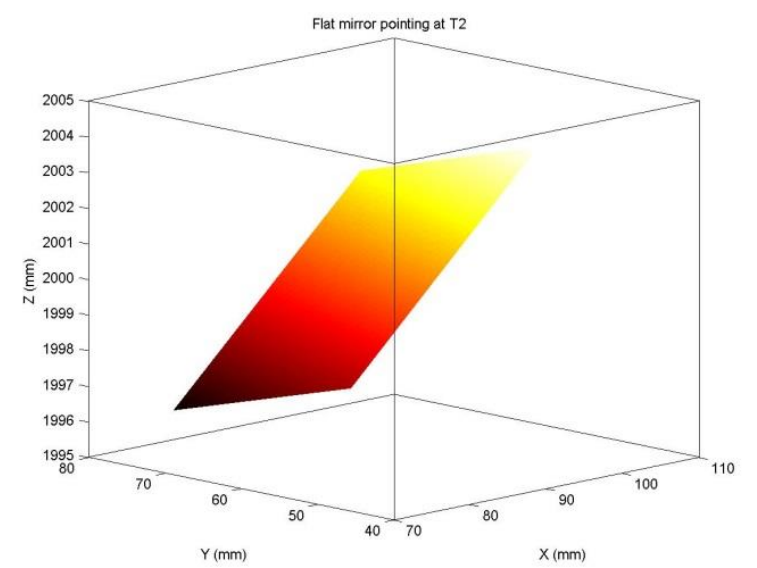

$10 c$

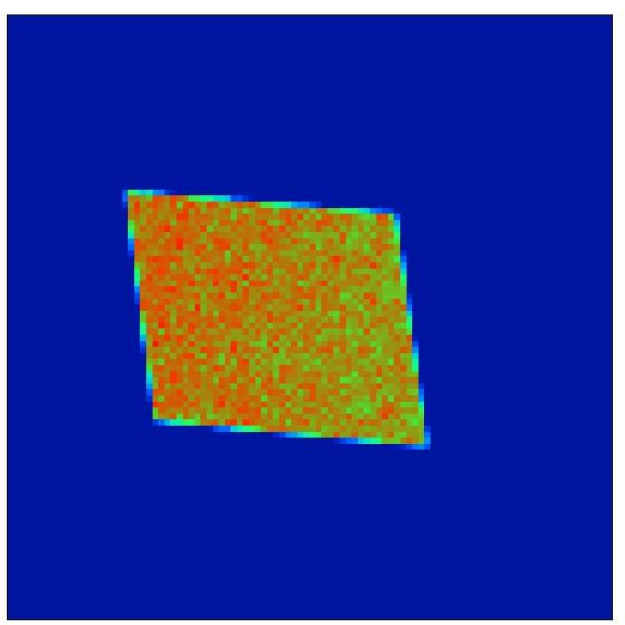

$10 \mathrm{~b}$

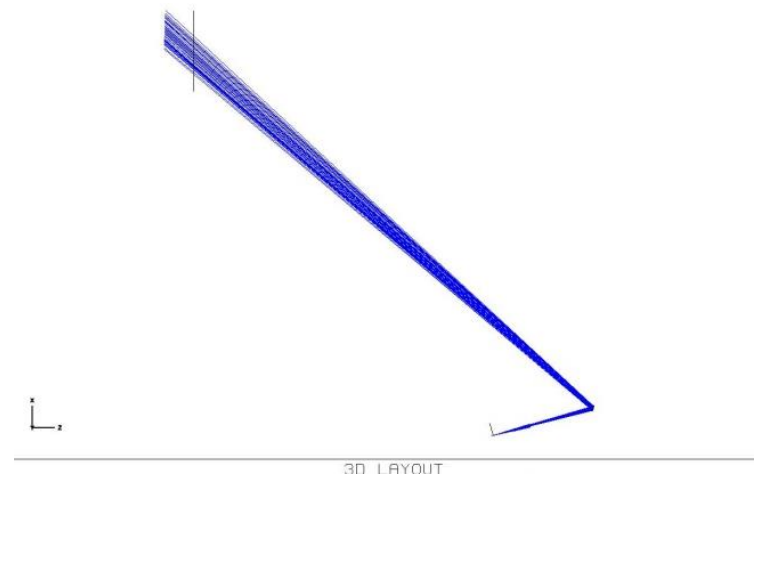

$10 \mathrm{~d}$

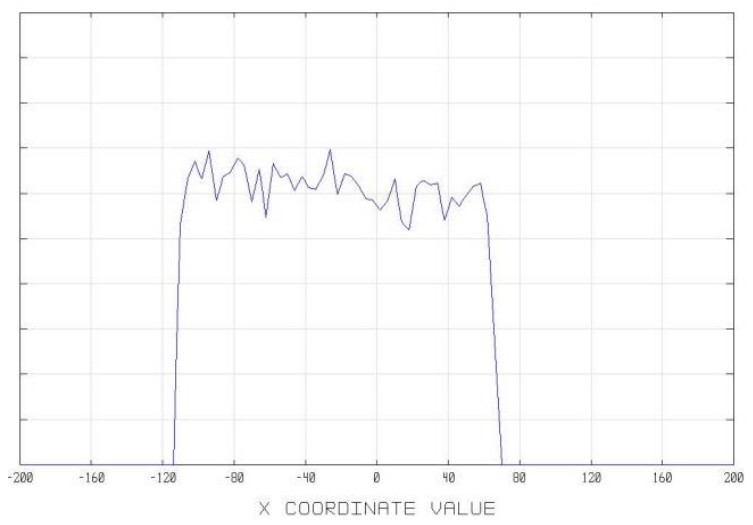

Figure 10: Flat reference mirror pointing at target T2.

Figure 10a: Surface of the reference flat mirror .

Figure 10b : Zemax layout

Figure 10c: Image obtained with Zemax simulation using $10^{6}$ ray. Size of the spot diagram $185 \times 161 \mathrm{~mm}$.

Figure $10 \mathrm{c}$ : $\mathrm{X}$ irradiance sections.

All distances in mm. Irradiance normalized at 1. 
$11 a$

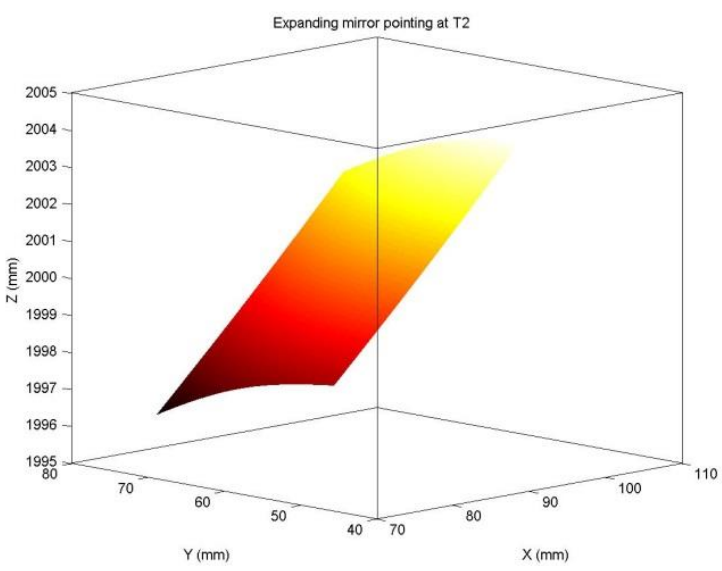

$11 \mathrm{c}$

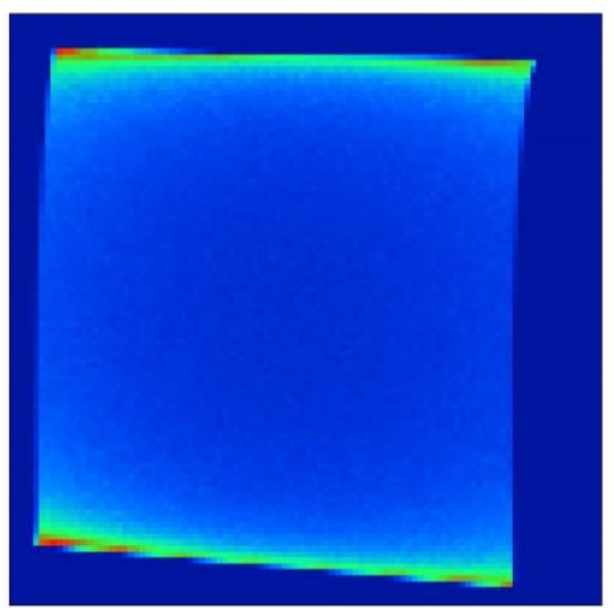

$11 b$

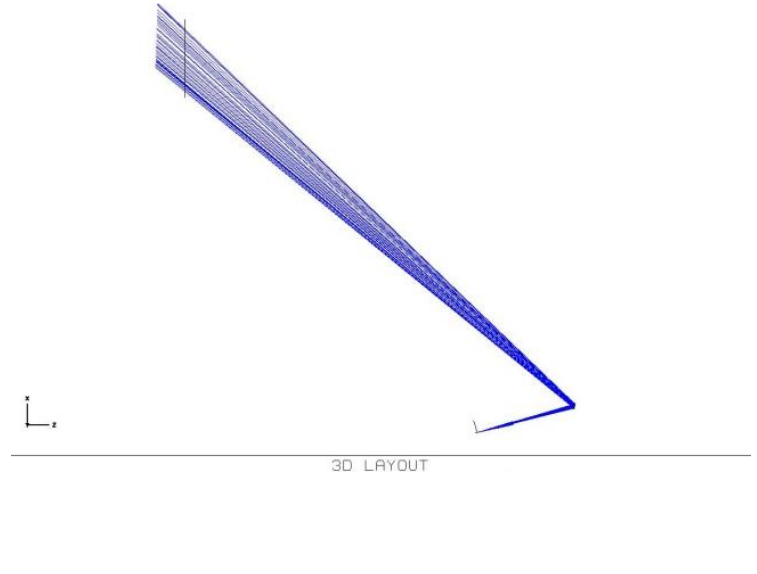

$11 d$

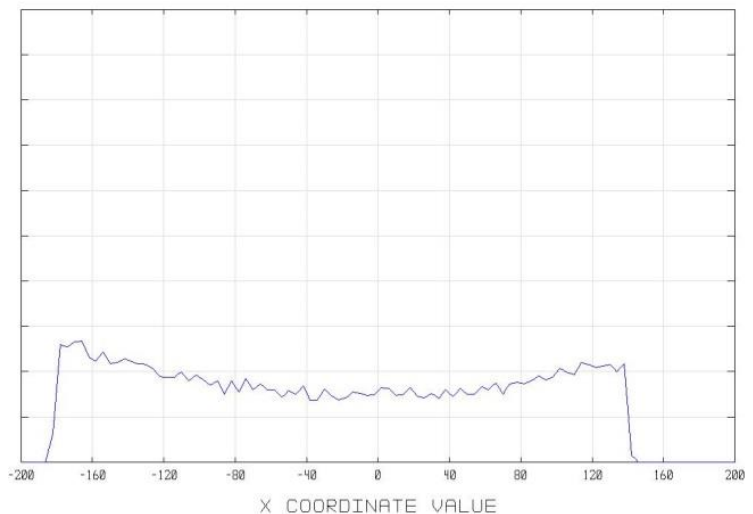

DETECTOR IMAGE: INCOHERENT IRRADIANCE

Figure 11 : Mirror obtained with an expansive merit function aiming at a $400 \times 400 \mathrm{~mm}$ squared patch illumination around target $\mathrm{T} 2$.

Figure 11a: Surface obtained after an elementary optimization process .

Figure 11b : Zemax layout

Figure 11c: Image obtained with Zemax simulation using $10^{6}$ ray. Size of the spot diagram $336.5 \times 353.5 \mathrm{~mm}$. Obtained size is $12.6 \%$ smaller than expected.

Figures 11d : X irradiance section.

All distances in mm. Irradiance normalized at 1 

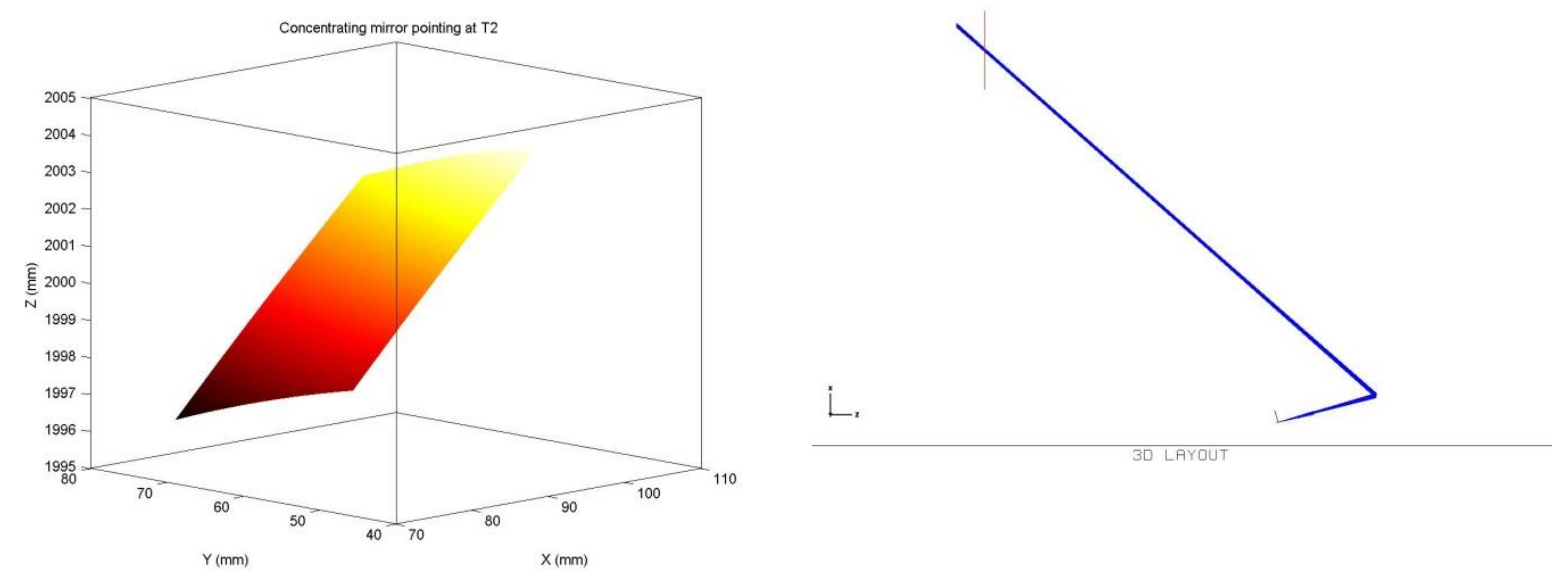

$12 \mathrm{c}$

$12 d$
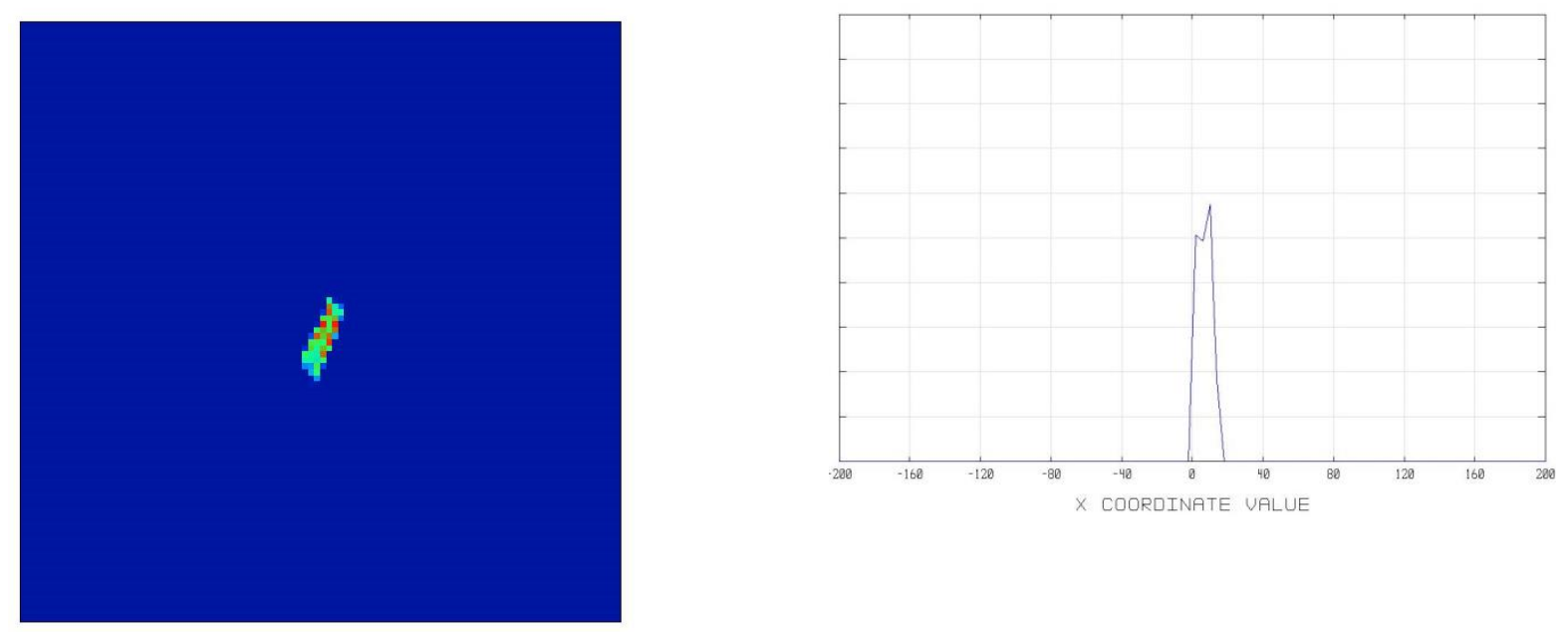

Figure 12 :Mirror obtained with a concentration merit function aiming to obtain the minimum spot around around target $\mathrm{T} 2$.

Figure $12 \mathrm{a}$ : Surface obtained after an elementary optimization process.

Figure 12b : Zemax layoutFigure $12 \mathrm{c}$ : Image obtained with Zemax simulation using $10^{6}$ ray. Size of the spot diagram $20 \times 43 \mathrm{~mm}$. Reduction size compared with the reference flat mirror is $76.8 \%$.

Figures $12 \mathrm{~d}$ and $12 \mathrm{~d}$ : $\mathrm{X}$ irradiance section.

All distances in mm. Irradiance normalized at 1.

\section{CONCLUSION}

The construction method for tailoring facets suitable for initial configurations to feed optimization processes is described in the general context of the design of reflectors for illumination. A detailed description on how to obtain the individual facets using bicubic Bézier surface descriptors is presented. 
The method describes how to find a Bézier surface for an arbitrary facet that best fits the proposed mapping process by departing from six points (source, target, and the four corners of the facet). This method is based on the selection of 6 strategic planes on which a 2D ray tracing will be applied. For each plane, 2D Bézier curves merged with elementary optimization and using a reduced number of rays will be obtained. A method to build up a 3D surface containing those curves is described.

For each facet, the final result is the description of a Bézier surface that can be transcribed as BSplines or NURBS, ready to be used with commercial CAD compatible tools.

This process can be applied to every facet. Indeed, our study describes a general initial configuration of the reflector by proceeding separately facet by facet.

To conclude, the process was tested in the context of an illumination system design process using a single facet submitted to two different conditions of tailoring and two different target positions, obtaining four different surfaces. The illumination distribution was simulated by Zemax and compared with those obtained with the corresponding reference flat mirrors pointing to the respective targets

\section{REFERENCES}

Ashdown I, Brackett WE, Sikkens M. 1994. Nonimaging Optics Design Using Genetic Algorithms. J. Illum. Eng. Soc. 23:12-21.

Baeuerle A, Bruneton A, Wester R, Stollenwerk J, Loosen P. 2012. Algorithm for irradiance tailoring using multiple freeform optical surfaces. Opt. Express 20.

Canavesi C, Cassarly WJ, Rolland JP. 2012. Direct calculation algorithm for two-dimensional reflector design. Opt. Lett. 37.

Cassarly WJ. 2007. Illumination merit functions - art. no. 66700K. Winston RJ RK, editor. Proc. Soc. PHOTO-OPTICAL Instrum. Eng. 6670.

Cassarly WJ, David SR, Jenkins DG, Riser AP, Davenport TL. 2000. Automated design of a uniform distribution using faceted reflectors. Opt. Eng. 39:1830-1839.

Cuevas F, Gonzalez O, Susuki Y, Hernandez D, Rocha M, Alcala N. 2006. Genetic algorithms applied to optics and engineering. In: Rosas E, Cardoso R, Bermudez JC, Barbosa-García O, editors. PROCEEDINGS OF THE SOCIETY OF PHOTO-OPTICAL INSTRUMENTATION ENGINEERS. SPIE-INT SOC OPTICAL ENGINEERING. p. 60461K-60461K-9.

Doyle S, Corcoran D, Connell J. 1999. Automated mirror design using an evolution strategy. Opt. Eng. 38:323-333. 
Fang Y-C, Tsai C-M. 2008. Miniature lens design and optimization with liquid lens element via genetic algorithm. J. Opt. A Pure Appl. Opt. 10:075304. Farin G. 2002. Curves and surfaces fot CAGD (5th edition). San Francisco: Morgan Kaufmann.

Fournier FR, Cassarly WJ, Rolland JP. 2010a. Fast freeform reflector generation using source-target maps. Opt. Express 18:5295-5304.

Fournier FR, Cassarly WJ, Rolland JP. 2010b. Freeform reflector design using integrable maps. Bentley J, Gupta A, Youngworth RN, editors. Proc. SPIE-The Int. Soc. Opt. Eng. 7652:765221.

Gur E, Mendlovic D, Zalevsky Z. 2002. Optical Generation of Fuzzy-Based Rules. Appl. Opt. 41:4753. Jester P, Menke C, Urban K. 2011. B-spline representation of optical surfaces and its accuracy in a ray trace algorithm. Appl. Opt. 50:822-828.

Kostrzewski AA, Kim DH, Jannson TP, Savant GD, Kim J, Chen J. 1998. Ongoing applications of soft computing technologies to real world problems at Physical Optics Corporation. In: Bosacchi B, Fogel DB, Bezdek JC, editors. APPLICATIONS AND SCIENCE OF NEURAL NETWORKS, FUZZY SYSTEMS, AND EVOLUTIONARY COMPUTATION. SAN DIEGO, CA: SPIE-INT SOC OPTICAL ENGINEERING. p. 170-173.

Leutz R, Ries H. 2003. Tailored Fresnel optics II - The facets. ISIP:000188513500019: SPIE-INT SOCIETY OPTICAL ENGINEERING (DESIGN OF EFFICIENT ILLUMINATION SYSTEMS Proceedings of the society of photo-optical instrumentation engineers (Spie)).

Magarill S. 2011. Skew-faceted elliptical reflector. Opt. Lett. 36:532-533.

Michaelis D, Schreiber P, Braeuer A. 2011. Cartesian oval representation of freeform optics in illumination systems. Opt. Lett. 36:918-920.

Oliker V. 2003. Mathematical aspects of design of beam shaping surfaces in geometrical optics. Kirkilionis M, Kromker S, Rannacher R, Tomi F, editors.

Oliker V, Newman E. 1993. The Energy-Conservation Equation in the Reflector Mapping Problem. Appl. Math. Lett. 6:91-95.

Pohl W, Anselm C, Knoflach C, Timinger AL, Muschaweck JA, Ries H. 2003. Complex 3DTailored facets for optimal lighting of facades and public places. Koshel RJ, editor. Proc. Soc. PHOTO-OPTICAL Instrum. Eng. 5186.

Ries H. 2011. Boundary conditions for balancing light in tailoring freeform surfaces. Kidger S. TED, editor. Proc. SPIE 8170:817002.

Ries H, Muschaweck J. 2001. Tailoring freeform lenses for illumination. ISIP:000174395600006: SPIE-INT SOCIETY OPTICAL ENGINEERING (NOVEL OPTICAL SYSTEMS DESIGN AND OPTIMIZATION IV Proceedings of the society of photo-optical instrumentation engineers (Spie)). 
Ries H, Muschaweck J. 2002. Tailored freeform optical surfaces. J. Opt. Soc. Am. A-Optics Image Sci. Vis. 19:590-595.

Sikkens M, Nuyens P. 2003. Structured design method for automotive lamp reflectors.

Mouroulis WJ Johnson,RB PZS, editor. Proc. Soc. PHOTO-OPTICAL Instrum. Eng. 5173.

Timinger A, Kribus A, Doron P, Ries H. 2000. Faceted concentrators optimized for homogeneous radiation. Appl. Opt. 39.

Turner MG, Garcia KJ. 2008. Optimization using rational Bezier control points and weighting factors. Koshel RJ, Gregory GG, Moore JD, Krevor DH, editors. Proc. Soc. PHOTO-OPTICAL Instrum. Eng. 7061:70610H-70610H.

Wu B-W, Liu T-K, Fang Y-C, Chou J-H, Tsai H-L, Chang E-H. 2008. Primary Chromatic Aberration Elimination via Optimization Work with Genetic Algorithm. In: Mazuray L, Wartmann R, Wood A, Tissot J-L, Raynor JM, editors. OPTICAL DESIGN AND ENGINEERING III, PTS 1 AND 2 Proceedings of SPIE. SPIE-INT SOC OPTICAL ENGINEERING. p. 71000J-71000J-15. [Xu O. 2011. Collimation Lens Design using AI-GA Technique for Gaussian Radiators with Arbitrary Aperture Field Distribution. J. Electromagn. Waves Appl. 25:743-754. Yang B, Wang Y, Liu Y, Sasian J, Koshel J. 2009. Efficient ray-tracing for free-form reflectors. Optik (Stuttg). 120:40-44.

Núria Tomás-Corominas is an assistant professor at the Universitat Politècnica de Catalunya. She received her BS in optics from Universitat Politècnica de Catalunya in 1990 and MS degree in Applied Optics from the Reading University in 2001. Her research activity focuses on Optical Design and she is preparing her PhD Degree in Physics at UPC. She is currently lecturer of several subjects related with Optometric Instrumentation in the BSc and MSc of the Terrassa School of Optics and Optometry (UPC) 